\title{
Los rituales funerarios, un acercamiento teórico y metodológico al estudio de las prácticas mortuorias y sus significados durante la fase Curridabat (300-800 d.C.)
}

\author{
Jeffrey Peytrequín Gómez*
}

\begin{abstract}
RESUMEN
En este artículo se discuten los alcances de un acercamiento teórico específico, basado en la perspectiva de la historia como una narrativa y la arqueología de acción, con respecto al análisis de contextos funerarios. La información se va hilando con el propósito de sugerir su aplicación hacia un caso de estudio específico referente a prácticas rituales en el Intermontano Central de Costa Rica.
\end{abstract}

Palabras clave: agencia, narrativa, rituales funerarios, habitus, Curridabat.

\begin{abstract}
This essay discusses the reaches of a specific theoretical approach, based on the perspective of the history as a narrative and the archaeology of action, with regard to the analysis of funeral contexts. The information is presented with the purpose of suggesting its application toward a specific study case of ritual practices in the Central Area of Costa Rica.
\end{abstract}

Keywords: agency, narrative, mortuary rituals, habitus, Curridabat.

\section{Introducción}

"Sólo cuando planteamos hipótesis acerca de los significados subjetivos presentes en la mente de una comunidad humana del pasado podemos empezar a hacer arqueología”

(Hodder, 1994:95).

El estudio de los contextos funerarios y las prácticas rituales ha sido uno de los focos de interés de la arqueología durante muchos años. Considerando la singularidad de las prácticas rituales funerarias de la Fase Curridabat, se intentará justificar la aplicación de un acercamiento teórico alternativo que toma en cuenta la cosmovisión y la acción humana como motor de cambio y expresión de ideas en el pasado.

\footnotetext{
* Jeffrey Peytrequín Gómez. Costarricense. Licenciado en Antropología con énfasis en Arqueología y candidato a Magíster en Antropología, Universidad de Costa Rica. Arqueólogo consultor independiente, odiseo@costarricense.cr
} 


\section{Alternativa teórica para el entendimiento de las prácticas rituales}

El cambio que en los estudios históricos ocasionó la Escuela de Annales ${ }^{1}$, en Francia, es fundamental para la comprensión de un enfoque que considera las ideas de las personas, antes que los fenómenos o estructuras inmanentes, las causas que rigen el destino de los seres humanos.

Los Annales se caracterizaron por un acercamiento multidisciplinario y por sobrepasar el énfasis tradicional en los estudios sobre la historia política y los "grandes hombres", además del rechazo por las nociones de "objetividad" para escribir la historia(s) y, por supuesto, el interés por las mentalités.

Las mentalités hacen alusión a la Historia de las ideologías, cosmovisiones y estructuras mentales (en otras palabras el contexto histórico) y no a la historia de las ideas (elitista).

Un importante exponente de esta escuela de pensamiento, Fernand Braudel, empezó a estudiar la historia de una región en términos de geografía humana y topografía. Asimismo, este autor estableció estructuras temporales de cambio histórico en tres niveles de particularidad (Braudel, 1976:352-353).

A pesar de que el enfoque de Braudel fue revolucionario para el estudio de la historia, ciertos aspectos del mismo han sido criticados:

$>$ 1. La falta de consideración de las mentalités, pensaba que el verdadero contexto histórico residía en un nivel estructural mucho más profundo.

2. Su enfoque tenía cargas de determinismo. Existe un concepto deficiente acerca de la agencia.

3. La estructuración geográfica se concibe como estática y no es susceptible a la intervención humana, aunque de hecho las prácticas humanas alteran significativamente el ambiente, inclusive dentro del periodo cubierto en su estudio del Mediterráneo (Last, 1995:142).

Los nuevos enfoques, a pesar de la crítica de la misma escuela de Annales, tendieron a cambiar la "objetividad" de las estadísticas de los estudios históricos por las estructuras narrativas. Los Annales temían que la narrativa hiciera que la historia tuviera una relación muy cercana con el estilo discursivo literario y, por lo tanto, se alejara de su "cientificidad".

Como reacción a lo anterior, filósofos de la historia como White (1992) y Ricoeur (1971) argumentaron que la narrativa es una parte integral del discurso histórico. Los siguientes estudios mostraron un interés en las mentalités y un tratamiento de los temas a nivel microhistórico y regional. Además, tuvieron un acercamiento importante hacia la antropología histórica, la

\footnotetext{
${ }^{1}$ Llamada así por el órgano de difusión de sus ideas, la revista Annales d'histoire économique et sociale, luego llamada Annales: économies, sociétés, civilisations fundada por Marc Bloch y Lucien Febvre en 1929.
} 
historia narrativa y política (e.g. biografía) y se empezó a poner en tela de duda la exactitud de la información de los registros.

Es así como una de las reacciones más importantes hacia la historia Braudeliana fue el mostrar que la agencia humana es necesaria para la explicación histórica; este redescubrimiento de la agencia intenta superar la visión "objetiva" de la historia.

\section{Aproximación narrativa a la historia antigua}

La cualidad procesual de la estructura de larga duración braudeliana (proceso histórico) fue de mucho interés para investigadores como Ian Hodder que vieron en ésta, una herramienta para superar la visión de explicaciones funcionales causales que caracterizaban a la Nueva Arqueología. La posibilidad de realizar un estudio con este enfoque apelaba a un proyecto postprocesual, permitiendo a la vez teorizar acerca de la interacción entre la estructura y los eventos.

Así, con una integración de perspectivas históricas y arqueológicas se puede llegar a un buen estudio del pasado. Como menciona Last (1995:144):

\section{"Específicamente, la arqueología puede brindar evidencia de naturaleza de estructuras de larga duración, permitiendo por un lado la investigación de la dialéctica entre las estructuras geográficas y socio- económicas y por el otro los eventos históricos".}

En este mismo sentido, Hodder (1990) relaciona ambas perspectivas y aduce que la narrativa estructura la vida de las personas de una forma análoga a la que los arqueólogos estructuran el registro arqueológico.

Este pensamiento está basado en autores como Ricoeur (1971) y White (1992) que, como se mencionó arriba, conciben la vida real estructurada como una narrativa y a la narrativa como un apropiado (y necesario) modo discursivo para la historia.

Lo que hace aceptable una historia desde este punto de vista es la forma y la coherencia, características dadas por la narrativa. Shanks y Tilley $(1994: 16,19)$ mencionan que el discurso de la historia, la producción de textos, es parte del mismo proceso de la historia. Aquí no se está considerando la narrativa histórica tradicional, sino una narrativa analítica y retrospectiva donde se ve el pasado desde el presente y no se pretende resolver cosas sino interpretarlas.

Los eventos históricos vistos de esta forma poseen la misma estructura que el discurso narrativo. El entendimiento del mundo y la historia está basada en hacer una alegoría semejante "posicionada hacia" la realidad, pero no es la realidad en sí. Este proceso tiene tres aspectos o momentos, los cuales serán discutidos más adelante con respecto al estudio de caso:

1. entender la acción humana y las estructuras temporales; 
2. entender la historicidad lograda cuando estos eventos son ubicados dentro de un contexto, lo cual significa el rol del argumento de enganchar varios factores dispares como son los eventos, los agentes, las metas, las consecuencias; y

3. la actualización del mundo presentado en el texto a través del acto de leer.

Estos tres aspectos son los mimeses discutidos por Hodder (1990). El uso de este término implica una clase de "imitación de lo real", una representación simbólica de los eventos reales que es realista porque los eventos reales son en sí simbólicos, apelando finalmente a un nivel de "profunda temporalidad" que fundamenta toda la historia. Según Shanks y Tilley (1994:21), este concepto fue acuñado por Aristóteles para referirse a la relación entre la realidad y la producción de un texto.

De esta forma, un texto mimético no copia o duplica la realidad pero sí la imita de forma creativa. Este tipo de textos no son duplicados objetivos de la realidad, ni tampoco fantasías subjetivas del "narrador". En este caso no serían invenciones antojadizas del arqueólogo; sino, más bien, juicios de probabilidad con base en los contextos arqueológicos y otra información complementaria (e.g. datos etnográficos) que sirva para darle coherencia a las posibles explicaciones.

En este argumento narrativo la explicación (y/o interpretaciones) no constituye la historia entera, solo puede ser vista como un medio para entender la unión de los eventos dentro de una estructura narrativa. La pregunta sobre la distinción entre la realidad histórica y su representación textual es irrelevante cuando aceptamos que el entendimiento es imposible fuera de una representación narrativa.

La verdad "del pasado" es en un sentido una metáfora; una metáfora es una figura que establece una identidad entre distintas cosas y los objetos. Es una producción de nuevos significados a través del descubrimiento de la similitud en la diferencia. Los arqueólogos encuentran su afinidad con el pasado a través de su distinción con éste, con prácticas que relacionan el pasado con el presente (Shanks y Tilley, 1994:20).

Como respuesta a este punto de vista se han dado una serie de críticas, entre las que tenemos las siguientes. En primer lugar, se ha expuesto que siempre los historiadores reflejan un discurso oculto, donde se aplican sus propias preconcepciones del mundo al análisis histórico. Con esto se ha apelado a que la narrativa es muy “subjetiva” y, por tanto, pierde su valor científico.

No obstante, debemos recordar que todo conocimiento es el reflejo de una situación específica, ya sea personal, de la línea de la Academia en la cual el investigador está inscrito, de la realidad político-social de su país, entre otros campos (Bourdieu 2000:13). Por lo que la construcción del mismo siempre está relativizada y la labor de los investigadores es hacer notar esos factores para poder comprender sus líneas de interpretación.

En palabras de Shanks y Tilley (1994:18), la narración es una reflexión y creación de un mundo donde la experiencia existe como un flujo y una continuidad, donde el significado y el tiempo 
están relacionados orgánicamente, donde la arqueología y la historia son una serie orgánica de eventos saturados de significado.

También se han criticado los enfoques donde la sociedad reduce los agentes a un epifenómeno de estructuras. Por ejemplo la consecuencia en una interpretación arqueológica, que derive de un enfoque así, es que la cultura material puede ser vista como una evidencia directa de las estructuras ideológicas, y se puede efectivamente ignorar los agentes individuales que la producen.

En otras palabras, sería darle una prioridad al relato sobre los protagonistas (una represión de los agentes). La teoría de la acción ha llenado estos vacíos. A continuación se comentará sobre cómo se concibe la agencia para su utilización en la interpretación arqueológica.

\section{La agencia como motor de cambio}

La agencia es un acto caracterizado por la volición y la intención y no puede ser reducido a meros eventos. Esto quiere decir que el acto es libre, seleccionado y pretende tener una incidencia en la práctica. Asimismo, toda consecuencia producto de una acción intencional puede ser el resultado de la agencia, ésta es la vía por la que la gente efectúa, o puede efectuar, un cambio.

La habilidad que poseen los agentes de actuar es la clave de su agencia, ya que no todas las personas tienen la misma capacidad de actuar sobre su sociedad. Es necesario aclarar que la agencia puede ser colectiva -a través de grupos de parentesco, etarios, entre otros- y no sólo referirse a la acción individual.

De hecho, la agencia incluye la acción de colectividades más allá que la de individuos separados y sus propias vidas. Además siempre está situada en condiciones estructuradas, que facilitan sus acciones, porque la agencia humana requiere un medio a través del cual funcionar.

Las prácticas sociales están estructuradas por ciertos recursos que son sus medios y su consecuencia. Estos recursos van desde cuestiones materiales y simbólicas, hasta tradiciones de ejecución y expresión que mantienen o transforman la estructura en tiempo y espacio (Barrett, 2002:149-150).

\section{La relación entre estructura y agencia}

Siempre hay restricciones prácticas en el rango de las opciones factibles de acción y éstas dependen del contexto social del individuo, este contexto es una manifestación específica que se puede denominar "estructura social".

Existen dos posiciones filosóficas extremas que conciben la relación entre la estructura social y los agentes: 
La primera es el objetivismo puro. Para esta postura la acción está sujeta a un determinismo causal por parte de estructuras preexistentes. Así, los factores externos reprimen de forma inaceptable la libertad individual, esto con las implicaciones políticas negativas (abuso de poder) del caso. En los estudios arqueológicos dichas estructuras inmanentes se han asociado a causas como la ecología, la economía o el mantenimiento del "sistema" como tal.

La corriente del subjetivismo puro incluye de forma principal las perspectivas existencialistas (e.g. Sartre). Aquí, a pesar de que se concibe la acción como un tipo de confrontación con el mundo y que las prácticas de los agentes están tanto habilitadas como reprimidas por redes sociales e ideológicas, el individualismo niega la fuerza de los intereses grupales y a las colectividades; así como el impacto de las estructuras pre-existentes donde los individuos socializan.

La solución de esta relación sería integrar estructura y agencia. Un autor muy importante como Bourdieu (1990a:64) dice que la práctica está condicionada por el "habitus", éste es un sistema de disposiciones estructuradas y durables producidas históricamente. Los habitus varían entre los individuos, pero el habitus individual está en una relación de homología. Estas disposiciones avalan y reprimen la acción en el nivel del conocimiento práctico, "esas cosas que son conocidas y no necesitan ser articuladas de forma conciente", por ejemplo el despliegue de ciertos rituales funerarios. Asimismo, la acción mediada por el habitus es instintiva y regulada.

Un acercamiento más dialéctico y auto-conciente hacia esta relación entre estructura y agencia es el propuesto por Anthony Giddens, su teoría de la estructuración. La estructuración es un conjunto de condiciones que intervienen en la estructura y la práctica para asegurar la reproducción o transformación de esa misma estructura (Giddens, 1984). En este esquema, la relación entre la agencia y la estructura es una "dualidad" (cada una está implicada en la producción de la otra) en vez de un "dualismo" (oposición categorial).

En la estructuración social los actores tienen reglas y accesos diferenciales a recursos en una diversidad de contextos de acción, los cuales se definen a través de la interacción, la producción y reproducción de las relaciones sociales.

Las acciones -al igual que en Bourdieu- se llevan a cabo en el nivel de una conciencia práctica, la cual no necesita ser articulada. La estructura siempre reproduce, por medio de la dualidad con la agencia, diferentes cualidades de conocimiento el cual opera a través de distintas formas de práctica social.

En esta teoría Giddens (1984) trata de darle más libertad a los agentes y el poder (relaciones de) es muy importante. El poder es generado en, y a través de, la reproducción de ciertas estructuras de dominio. Asimismo, una acción exitosa implica la habilidad (de los agentes) para movilizar recursos. Los agentes pueden actuar para mantener o transformar las estructuras, pero ello siempre depende del acceso diferencial a los recursos (e.g. simbólicos) y de su propia competencia práctica. 
Sobre el punto de vista de Giddens han surgido varias críticas a considerar. Por ejemplo, autores como Shanks y Tilley (1987:212) han mencionado que su particular preocupación por la conciencia práctica y la rutina le da mucho énfasis, en su estructuración, a la probabilidad de la estabilidad social. Lo cual, dentro de la arqueología, no da cabida a la percepción del cambio cultural en sí.

Esta racionalidad consistente y totalizadora parece que asume que la naturaleza humana esencialmente no ha cambiado y no se sugiere cómo o cuándo el "agente racional" fue generado, ni permite ver los cambios en la agencia.

En suma, la estructura es tanto el medio como el resultado de la acción. El individuo ostenta un rol fundamental como elemento autorresponsable, creativo y con grados distintos de competencia. La cultura material en concreto desempeña un rol muy activo creando la sociedad, así como un cambio continuo en ésta (Hodder, 1994:90).

\section{Discurso de conciliación- aplicabilidad al estudio de caso: los rituales funerarios durante la Fase Curridabat (300-800 d.C.)}

Acerca de la narrativa Shanks (referido por Last, 1995:153) menciona que:

"los relatos históricos también hablan ... del dolor humano de la historia. La historia no es simplemente un acto intelectual de construcción y creación, necesita de la poesía y del reconocimiento de la muerte y el decaimiento, que son el corazón de la historia”.

Si tomamos en consideración que el foco de interés serían las prácticas rituales funerarias en el Valle Central durante la Fase Curridabat, es de vital importancia la relación existente entre estos rituales y la muerte. Una relación ideológica entre el grupo social, el difunto, la muerte, el acto de enterramiento y las prácticas relacionadas a éste.

Los aspectos afectivos y a-estéticos de los artefactos (e.g. ofrendas funerarias) cuentan historias humanas, relatos ideológicos que nos permiten criticar nuestros conceptos y presupuestos. En palabras de Hodder (1995:168), la narrativa actúa en la experiencia práctica produciendo y "refigurando" nuestra propia experiencia temporal.

Así, la evidencia arqueológica permite una "traducción" entre el pasado y el presente. La narrativa, por ende, nos da la oportunidad de hacer interpretaciones diferentes a las tradicionales pues se enfoca (entre otros aspectos) en la(s) ideología(s) que hay detrás, en este caso, de un ritual funerario específico.

Con respecto a los tres puntos discutidos como ejes de la formación del discurso narrativo debemos mencionar que:

Con relación a entender la acción humana y las estructuras temporales, se debe hacer referencia a la contextualización de la actividad como tal y su localización espacio- temporal. Esto sería la 
caracterización de los espacios rituales funerarios, ¿qué se está entendiendo por contexto funerario?. O sea, hablar sobre las particularidades de los enterramientos tanto a nivel intra sitio como a una escala mayor; que incluya todos los sitios de una muestra particular ${ }^{2}$.

Lógicamente, los anteriores contextos serían discutidos en razón de compartir una temporalidad específica (e.g. Fase cultural) y cierta correlación espacial; en específico, el Intermontano Central de Costa Rica.

El segundo aspecto es entender la historicidad lograda cuando los eventos son ubicados dentro de un contexto general. Ya definidas las prácticas a estudiar -los rituales funerarios de la Fase Curridabat-, habría que relacionarlas con algunos aspectos de otras Fases culturales.

En otras palabras, es necesario evaluar las manifestaciones rituales que caracterizan al espacio temporal 300-800 d.C. Por ejemplo, se podría discutir sobre la continuidad cultural (un tipo de enculturación según Bourdieu) que ocurre, desde la Fase Pavas (300 a.C.-300 d.C.), con el uso de figuras aplicadas en el hombro de los soportes de las vasijas; hasta su gran expresión estilística y ritual en Curridabat.

Por otro lado, es importante destacar las diferencias entre los procedimientos mortuorios de distintas Fases. Entonces, ¿qué pudo significar el hecho de que durante Curridabat y la Fase Cartago (800-1500 d.C.) existan variantes en cuanto al acomodo del(os) difunto(s), la disposición de ofrendas ${ }^{3}$, o la conformación de las tumbas?.

Las respuestas a esta clase de preguntas deben apuntar hacia concepciones particulares sobre la muerte; y a las identidades grupales establecidas alrededor de ellas.

Así, el argumento engancharía varios de los llamados factores dispares como son los eventos (el ritual o los rituales), los agentes (las personas actuando y formando parte de esas prácticas), las metas (ideología-cosmovisión, intenciones) y las consecuencias (¿reconocimiento social?, ¿identidad colectiva?, ¿herencias?, ¿duelo?, entre otras).

Por último, se hilarían todos los aspectos anteriores. Se crearía un discurso narrativo para su presentación; se interpretaría el mundo, plasmado en el texto, a través del acto de leer. Aquí, se daría una(s) interpretación(es) a partir de la experiencia, el contexto y el punto de vista del investigador. Asimismo, cada persona al leer el argumento puede hacer nuevas interpretaciones de las prácticas rituales funerarias.

Cabe decir que las prácticas rituales están integradas por una serie de símbolos, los símbolos son fragmentos arbitrarios de la realidad incorporados en una experiencia fenomenológica. Así vistos, los símbolos constituyen un reino específico de la vida cultural (Robb, 1998:334). Además, el significado no reside en los artefactos como tal o en las personas que los utilizaron,

\footnotetext{
${ }^{2}$ En este caso, los sitios funerarios en el Valle Central con una temporalidad afín a la Fase Curridabat (300-800 d.C.).

${ }^{3}$ No sólo la ubicación, también la cantidad y variabilidad del ajuar.
} 
sino en el momento de interacción entre ambos (Thomas, 1996:97); o sea, en las prácticas rituales funerarias.

A este respecto, Bourdieu (1990b:861) menciona que:

“los sistemas simbólicos le deben su coherencia práctica ..... al hecho de que son el producto de prácticas que solo pueden satisfacer sus funciones prácticas en cuanto se lleven a cabo, son principios que no son solo coherentes -son capaces de generar prácticas que al mismo tiempo son coherentes intrínsicamente y compatibles con las condiciones objetivassino también prácticos, en el sentido de la conveniencia...”.

En un análisis de este tipo importarán las ofrendas no per se, no por el hecho de estar en el contexto arqueológico (funerario). Las ofrendas tendrán sentido en el punto de que su presencia, posición, cantidad, etc., posean una coherencia argumentativa con la práctica ideológica; con una cosmovisión particular de la muerte.

Por ejemplo, al saber que ciertos utensilios -a partir de su forma- pudieron servir para la ingesta de líquidos (e.g. jarrones trípodes, tazones); es necesario hacer algunas preguntas relacionadas con el contenido del significado del contexto, como:

¿Por qué se consumían líquidos en el ritual funerario?, ¿qué líquidos se ingerían?, ¿qué relación ideológica hay entre esos líquidos y la cosmovisión del grupo social?, ¿todos los agentes podían consumir esas bebidas?.

Estos y otros cuestionamientos nos permitirían acercarnos a la praxis del ritual, y concebir a los restos culturales como activos. Porque como menciona Bourdieu (1990a), aunque el habitus se constituye en la práctica; éste es objetivado en la cultura material y en los rituales.

Es importante anotar que algunos autores como Hodder (1994:24) y Robb (1998:338) han hecho la observación de que trabajos con este enfoque, por sus implicaciones, deben ser detallados estudios a pequeña escala. Esto con el fin de examinar el vínculo existente entre el individuo, los acontecimientos constituidos significativamente y las estructuras a largo plazo.

\section{A guisa de conclusión}

La narrativa es una forma necesaria en la explicación histórica. Debe ser expuesta como ideológica, sin embargo, constituye un modelo de "realidad" el cual nos permite apelar a las "pistas", los documentos y los artefactos arqueológicos. Lo anterior mantiene la diferencia entre la historia y la ficción. Es una teoría de la producción de textos escritos.

De esta forma, la narrativa posee una serie de mecanismos que permite darle sentido a una importante cantidad de información que, de ser otro abordaje, no se consideraría como "objetiva" o "científica" para la explicación de procesos relacionados a sociedades pretéritas. Esta clase de 
información se refiere a las intenciones e ideologías que son intrínsecas a las acciones tomadas por las personas en el pasado.

Con respecto a lo anterior, Shanks y Tilley $(1994: 19,21)$ dicen que los eventos tienen significado sólo y cuando están incorporados en textos, los cuáles, a su vez, deben tener sentido para una audiencia; deben ser incorporados en historias.

El tipo de narrativa que se pretende producir es aquella que rompa con la historia vista como una continuidad y que niega el cambio, las diferencias y las ambiguiedades propias de las relaciones sociales tanto presentes como las acaecidas en el pasado.

De tal manera, la producción de textos debe tener un juicio de valor; éste dependerá del punto de vista del "narrador". Al igual que una metáfora, el pasado requiere una interpretación.

En el mismo sentido, Robb (1998:338) menciona que esta arqueología es epistemológicamente más formulación que descubrimiento, interpretación de un texto en lugar del análisis de datos, plausibilidad en vez de prueba.

La naturaleza del concepto de habitus hace que éste sea muy útil para los arqueólogos. Quizá, las prácticas rituales funerarias de la Fase Curridabat sean un habitus regional.

La reconstrucción de los significados históricos de dichas prácticas debe basarse en argumentaciones de coherencia (narrativa) y de correspondencia con los datos empíricos. El presente ejercicio es solo un tipo de aproximación al tema, que forma parte de un continuo debate y para nada da por terminado el mismo.

\section{Literatura consultada}

Barrett, J. C. (2002). "Agency, the duality of structure, and the problem of the archaeological record”, en Hodder, I. (ed.) Archaeological theory today. Malden: Polity Press. pp. 141-164.

Braudel, F. (1976). El mediterráneo y el Mundo mediterráneo en la época de Felipe II. Madrid: Fondo de Cultura Económica.

Bourdieu, P. (1990a). The logic of practice. Standford: Standford University Press.

----. (1990b). Sociología y Cultura. México: Grijalbo.

----. (2000). "El campo científico", en Los usos sociales de la ciencia. Buenos Aires: Ediciones Nueva Visión. pp. 11-37.

Giddens, A. (1984). La constitución de la sociedad: contorno de la teoría de la estructuración. Cambridge: Polity Press.

Hodder, I. (1990). The Domestication of Europe. Oxford: Blackwell. 
-----. (1994). Interpretación en Arqueología: corrientes actuales. Segunda Edición (ampliada y puesta al día). Barcelona: Crítica.

----. (1995). "Material culture in time", en Hodder, I. et al. (eds.) Interpreting Archaeology: Finding Meaning in the Past. Nueva York: Routledge. pp. 164-168.

Last, J. (1995). "The nature of history", en Hodder, I. et al. (eds.) Interpreting Archaeology: Finding Meaning in the Past. Nueva York: Routledge. pp. 141-163.

Ricoeur, P. (1971). "The model of the text: meaningful action considered as a text", en Social Research. Vol. 38. pp. 529-562.

Robb, J. (1998). “The Archaeology of Symbols”, en Annual Reviews of Anthropology. Vol. 27. pp. 329-346.

Shanks, M. y C. Tilley (1987). Social Theory and Archaeology. Cambridge: Polity Press.

----. (1994). Re-constructing Archaeology. Theory and Practice. Londres: Routledge.

Thomas, J. (1996). Time, Culture and Identity. Londres: Routledge.

White, H. (1992). El contenido de la forma, narrativa, discurso y representación. Barcelona: Paidós. 